\title{
Transport Simulation for Helical Plasmas by use of Gyrokinetic Transport Model ${ }^{*}$
}

\author{
Shinichiro TODA, Motoki NAKATA, Masanori NUNAMI, Akihiro ISHIZAWA ${ }^{1)}$, \\ Tomo-Hiko WATANABE ${ }^{2)}$ and Hideo SUGAMA \\ National Institute for Fusion Science, NINS, 322-6 Oroshi-cho, Toki, Gifu 509-5292, Japan \\ ${ }^{1)}$ Graduate School of Energy Science, Kyoto University, Gokasho, Uji, Kyoto 611-0011, Japan \\ ${ }^{2)}$ Department of Physics, Nagoya University, Furo-cho, Nagoya, Aichi 464-8602, Japan
}

(Received 26 December 2018 / Accepted 21 February 2019)

\begin{abstract}
Transport simulation for the electron and ion temperature profiles is performed in helical plasmas by using the heat diffusivity models and the quasilinear flux models [S. Toda et al., Phys. Plasmas 26, 012510 (2019)] for the electron and ion heat turbulent transport. The turbulent transport for the nonlinear simulation results can be evaluated by these models. The high- $T_{i}$ and low- $T_{i}$ plasmas for the discharge in the Large Helical Device (LHD) are studied, where the ion temperature gradient mode is unstable. The electron and the ion temperature profiles of the dynamical simulation results do not contradict with those of the experimental results in the LHD. For the plasmas of the LHD in this study, the transport simulation results by the diffusivity models and the quasilinear flux models for the heat transport to reproduce the nonlinear simulation results in the allowable errors are found to explain the experimental results for the temperature profiles.
\end{abstract}

(C) 2019 The Japan Society of Plasma Science and Nuclear Fusion Research

Keywords: dynamical simulation, transport model, zonal flow, gyrokinetic simulation, turbulence, helical plasma

DOI: $10.1585 /$ prr. 14.3403061

\section{Introduction}

A quantitative prediction of turbulent transport $[1,2]$ is one of the most important issues to realize magnetic fusion energy. Recently, a large number of gyrokinetic simulations of the turbulent transport in toroidal plasmas have been performed [3-9]. The gyrokinetic analysis results in tokamak [10-12] and helical $[13,14]$ plasmas have been studied with the experimental results. In tokamak plasmas, the transport simulation, which is directly coupled to gyrokinetic analyses, is globally performed $[15,16]$. The gyrokinetic simulation for helical plasmas consumes much larger computer resources than those for tokamak plasmas, because the former requires a large number of mesh points along field lines to resolve helical ripple structures. Since it is still not easy to couple the nonlinear gyrokinetic simulation with an integrated transport simulation code for helical plasmas [17], the predictive model, which can quickly reproduce the nonlinear simulation results, is highly demanded.

The modeling of the turbulent transport in helical plasmas has been advanced. The gyrokinetic local flux tube code GKV code [18] has been used to investigate the ion temperature gradient (ITG) mode and zonal flows in the Large Helical Device (LHD) for studying the turbulent transport [13]. The gyrokinetic simulation in the adia-

author'se-mail: toda@nifs.ac.jp

*) This article is based on the presentation at the 27th International Toki Conference (ITC27) \& the 13th Asia Pacific Plasma Theory Conference (APPTC2018) batic electron condition is performed for the high and low ion temperature plasmas in the LHD of the shot number 88343 [19]. The reduced model for the ion heat diffusivity was constructed [14] by the simulation for the adiabatic electron approximation. This reduced model is the function of the linear growth rate for the ITG mode and the zonal flow decay time [20,21]. The ion heat diffusivity model for the kinetic electron response was shown in helical plasmas [22]. The heat diffusivity models for the electron and ion heat transport, and the quasilinear flux models for the particle and heat transport have been proposed, where the larger number of the wavelength in the wider wavelength region than those in [22] is taken [23]. How to apply the reduced model of the turbulent ion heat diffusivity in the adiabatic electron condition to the transport code has been shown in helical plasmas [24]. It is a challenging task to perform dynamical transport simulation by using the gyrokinetic transport model due to the wide range of the ion turbulence time scale for the gyrokinetic simulation $(10 \mu \mathrm{s})$ and the time scale for the transport simulation $(\sim 100 \mathrm{~ms})$ in the LHD.

In this study, the dynamical transport simulation is performed in helical plasmas by use of two kinds of the reduced models for the kinetic electron response (heat diffusivity and quasilinear flux models). The ion heat diffusivity model is installed into the integrated transport code for simulating evolutions of the plasma profiles in the LHD when the additional modeling by the normalized charac- 
teristic length for the ion temperature gradient, $R / L_{T_{i}}$ is used [24]. The additional modeling of the quantity related with the mixing length estimate and the zonal flow decay time in the diffusivity models, which are the linear gyrokinetic simulation results, is explained in Sec. 3. Here, $R$ is the major radius, $L_{T_{i}}=-T_{i} / T_{i}^{\prime}$ and the prime denotes the radial derivative. Electron heat diffusivity model is adopted to the transport simulation for the additional modeling by $R / L_{T_{i}}$. Electron quasilinear flux model is also installed to the transport simulation codes by the ratio of the electron quasilinear heat flux to the ion quasilinear heat flux, where the ion heat flux is evaluated by the heat diffusivity model. In the high- $T_{i}$ and low- $T_{i}$ plasmas, the ion temperature profiles of the dynamical transport simulation by use of the ion heat diffusivity model for the kinetic electron response are compared with the LHD experimental result. In the low- $T_{i}$ plasmas, the magnetic field configuration is shifted further inward than in the high- $T_{i}$ plasmas. The generation of zonal flows can be enhanced in the inward shifted configuration [25]. The electron temperature profiles of the transport simulation results by the electron heat diffusivity model and the quasilinear flux model are also compared with the experimental observation result in the LHD.

\section{Heat Diffusivity and Quasilinear Flux Models}

In this section, the electron and ion heat diffusivity models and the quasilinear models for the particle and heat transport are shown to reproduce the nonlinear gyrokinetic simulation results [23]. These models are constructed for the high- $T_{i}(t=2.2 \mathrm{~s})$ and low- $T_{i}(t=1.8,1.9 \mathrm{~s})$ plasmas in the LHD shot number 88343 [19]. The ITG mode is unstable in this article, because the real frequency is negative and the mode rotates towards the ion diamagnetic direction.

The electron and ion heat diffusivity models are represented by

$$
\frac{\chi_{e}^{\text {model }}}{\chi_{i}^{G B}}=\frac{A_{1 e} \mathcal{L}^{B_{1 e}}}{A_{2 e}+\tilde{\tau}_{Z F}^{B_{2 e}} / \mathcal{L}^{B_{3 e}}}
$$

and

$$
\frac{\chi_{i}^{\text {model }}}{\chi_{i}^{G B}}=\frac{A_{1 i} \mathcal{L}^{B_{1 i}}}{A_{2 i}+\tilde{\tau}_{Z F}^{B_{2 i}} / \mathcal{L}^{B_{3 i}}}
$$

where $\chi_{i}^{G B}\left(=\rho_{i}^{2} v_{t i} / R\right)$ is the gyroBohm diffusivity, $\mathcal{L}\left(\equiv \int\left(\tilde{\gamma}_{\tilde{k}_{y}} / \tilde{k}_{y}^{2}\right) d \tilde{k}_{y}\right)$ is the quantity related with the mixing length estimate and $\tilde{\tau}_{Z F}\left(=\tau_{Z F} /\left(R / v_{t i}\right)\right)$ is the normalized zonal flow decay time $[20,21]$. Here, $\tilde{\gamma}_{\tilde{k}_{y}}(=$ $\left.\gamma_{\tilde{k}_{y}} /\left(v_{t i} / R\right)\right)$ is the normalized linear growth rate of the ITG mode, $\tilde{k}_{y}\left(=k_{y} \rho_{i}\right)$ is the normalized poloidal wavelength, where $v_{t i}\left(=\sqrt{T_{i} / m_{i}}\right)$ is the ion thermal velocity and $\rho_{i}$ $\left(=m_{i} v_{t i} /(e B)\right)$ is the ion gyroradius. The coefficients are given by $A_{1 e}=1.3 \times 10, A_{2 e}=2.0, A_{1 i}=2.6 \times 10^{2}$, and $A_{2 i}=1.8 \times 10$. The exponents are shown by $B_{1 e}=0.30$,
$B_{2 e}=0.62, B_{3 e}=0.63, B_{1 i}=0.66, B_{2 i}=3.1$, and $B_{3 i}=0.26$. The coefficients in the ion heat diffusivity model (2) are different from those in [22], because the simulations are performed in the high- $T_{i}$ and low- $T_{i}$ plasmas for the discharge \#88343 in this article and only in the high- $T_{i}$ plasmas for [22]. With the much lower computational cost, the heat diffusivity models enable us to reproduce the nonlinear simulation results by use of linear simulation results.

The quasilinear flux models for the particle and heat transport are shown as

$$
\tilde{\Gamma}_{q l}^{\text {model }}=C_{\Gamma} \int \frac{\tilde{\Gamma}_{\tilde{k}_{y}}^{\text {lin }}}{\left\langle\left|\tilde{\phi}_{\tilde{k}_{y}}^{l i n}\right|^{2}\right\rangle}\left\langle\left|\tilde{\phi}_{\tilde{k}_{y}}\right|^{2}\right\rangle^{\text {model }} d \tilde{k}_{y},
$$

and

$$
\tilde{Q}_{j, q l}^{\text {model }}=C_{Q_{j}} \int \frac{\tilde{Q}_{j, \tilde{k}_{y}}^{l i n}}{\left\langle\left|\tilde{\phi}_{\tilde{k}_{y}}^{l i n}\right|^{2}\right\rangle}\left\langle\left.\tilde{\phi}_{\tilde{k}_{y}}\right|^{2}\right\rangle^{\text {model }} d \tilde{k}_{y}
$$

for the species $j$, where the quantities with the superscripts lin represents the linear simulation result. Here, the tilde represents the normalization of the energy and particle fluxes by the values of $n T_{i} v_{t i} \rho_{i}^{2} / R^{2}$ and $n v_{t i} \rho_{i}^{2} / R^{2}$, respectively. The coefficients $C_{Q_{e}}=0.78, C_{Q_{i}}=0.58$, and $C_{\Gamma}=0.73$. The quasilinear fluxes are proportional to the the product of the linear response function and the nonlinear electrostatic potential fluctuation. The model function with the mixing length estimate and the zonal flow decay time

$$
\left\langle\left|\tilde{\phi}_{\tilde{k}_{y}}\right|^{2}\right\rangle^{\text {model }}=\frac{C_{q 1}\left(\tilde{\gamma}_{\tilde{k}_{y}} / \tilde{k}_{y}^{2}\right)^{\alpha_{q 1}}}{C_{q 2}+\tilde{\tau}_{Z F}^{\alpha_{Z F}} /\left(\tilde{\gamma}_{\tilde{k}_{y}} / \tilde{k}_{y}^{2}\right)^{\alpha_{q 2}}} .
$$

The parameters are determined as $C_{q 1}=1.0 \times 10^{2}, C_{q 2}=$ $9.2 \times 10^{-4}, \alpha_{q 1}=0.54, \alpha_{q 2}=0.12$ and $\alpha_{Z F}=1.6$. For the particle and heat transport, the linear simulation results of the quantity related with the mixing length estimate and the zonal flow decay time reproduce the nonlinear simulation results by the quasilinear flux models.

In Sec. 5, the transport simulation for $T_{e}$ and $T_{i}$ is performed by the heat diffusivity and quasilinear flux models. The radial profile of $T_{i}$ is predicted only using the ion heat diffusivity model (2). The radial profile of $T_{e}$ is predicted using the electron heat diffusivity model (1) and the quasilinear flux model for the electron heat transport (4). The value of $\tilde{Q}_{e, q l}^{\text {model }}$ is obtained from the ratio of $\tilde{Q}_{e, q l}^{\text {model }} / \tilde{Q}_{i, q l}^{\text {model }}$, where $\tilde{Q}_{i, q l}^{\text {model }}$ is evaluated by the ion heat diffusivity model (2).

\section{Additional Modeling of Turbulent Heat Diffusivities}

In this section, how to install the heat diffusivity models is explained to a transport simulation. This method is same as the case for the adiabatic electron [24]. The 


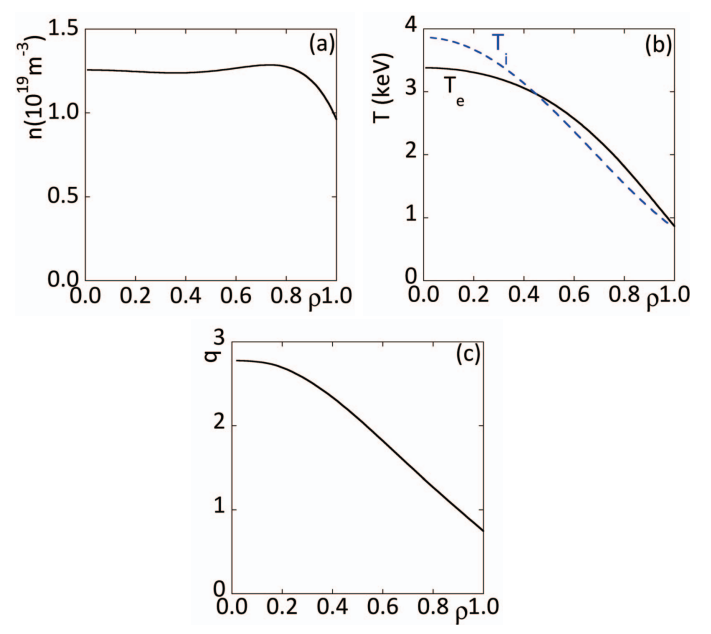

Fig. 1 The radial profiles of (a) the density, (b) the electron and ion temperatures and (c) the safety factor are shown for the high- $T_{i}$ plasmas.

linear analysis is done using the GKV code for the additional modeling of the turbulent heat diffusivities, before the dynamical transport simulation. For reducing a computational cost, the additional modeling is needed for $\mathcal{L}$ in terms of the physical parameter which is included in the transport codes. The characteristic length of the ion temperature gradient is considered to be the important parameter for the ITG instability. The parameter $L_{T_{i}}$ is considered to be more sensitive to the electron and ion heat fluxes than the electron temperature gradient scale length for the ITG mode studied here. As the function of the ion temperature gradient scale length $L_{T_{i}}$, the parameter $\mathcal{L}$ is additionally modeled by

$$
\mathcal{L}=a(\rho)\left(\frac{R}{L_{T_{i}}}-\frac{R}{L_{T_{c}}}\right),
$$

where $L_{T_{c}}$ is the normalized critical ion temperature gradient for the ITG instability and $\rho$ is the radial position normalized by the minor radius.

To find the critical ion temperature gradient, the dependence of $\mathcal{L}$ on $R / L_{T_{i}}$ is examined [8] with all plasma parameters fixed except the ion temperature gradient in the region $0.14 \leq \rho \leq 0.80$. When we examine the linear growth rate of the ITG mode, the radial profiles of (a) the density $(n),(\mathrm{b})$ the electron $\left(T_{e}\right)$ and ion temperatures $\left(T_{i}\right)$ and (c) the safety factor $(q)$ are used in Fig. 1 for the high- $T_{i}$ plasmas. Figure 2 shows the radial profiles of (a) the density, (b) the electron and the ion temperature, and (c) the safety factor for the low- $T_{i}$ plasmas. The electron temperature profile is same as the ion one for the low- $T_{i}$ plasmas. This simulation is performed for the three dimensional equilibrium field configuration with $R=3.75 \mathrm{~m}$ (the high- $T_{i}$ plasmas) and $R=3.6 \mathrm{~m}$ (the low- $T_{i}$ plasmas), using the plasma profiles explained here in the VMEC calculation. The slope $a(\rho)$ and the critical ion temperature gradient $L_{T_{c}}$ depend on the values of $L_{T_{e}}\left(=-T_{e} / T_{e}^{\prime}\right)$, $L_{n}\left(=-n / n^{\prime}\right)$ and the safety factor, which change due to
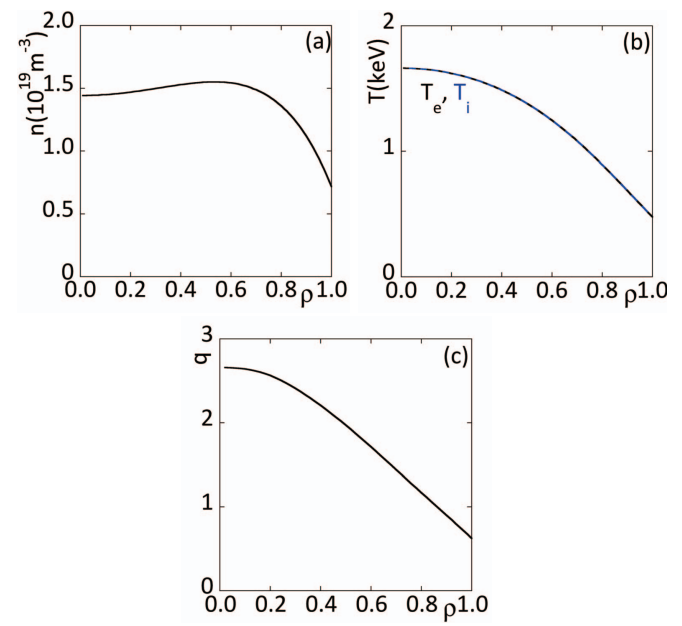

Fig. 2 The radial profiles of (a) $n$, (b) $T_{e}$ and $T_{i}$, and (c) $q$ are used for the low- $T_{i}$ plasmas.
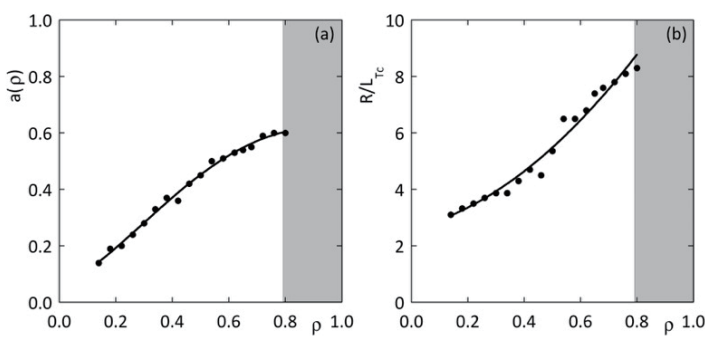

Fig. 3 The radial dependence of (a) a $(\rho)$ and (b) $R / L_{T_{c}}$ is shown with the filled circles for the high- $T_{i}$ plasmas. The curves represent the fitting function with respect to the radial position $\rho$.

the radial positions. We calculate the linear fitting function (6) at each radial point and obtain the critical values of $R / L_{T_{i}}, R / L_{T_{c}}$, where $\mathcal{L}$ becomes zero. The slope (a) $a(\rho)$ in terms of $R / L_{T_{i}}$ and the critical ion temperature gradient, (b) $R / L_{T_{c}}$ are obtained in Fig. 3 (the high- $T_{i}$ plasmas) and Fig. 4 (the low- $T_{i}$ plasmas). In the dynamical simulation, the plasma profile, for example, the ion temperature or the electron temperature, is fixed at the initial state in shaded region $0.8<\rho<1.0$. When we calculate the value of the ion heat diffusivity in the integrated transport code, the fitting functions of $a(\rho)$ and $R / L_{T_{c}}$ are used as $a(\rho)=0.19-0.96 \rho+6.6 \rho^{2}-9.4 \rho^{3}+4.3 \rho^{4}$ and $R / L_{T c}=2.6+2.5 \rho+6.5 \rho^{2}$ for the high- $T_{i}$ plasmas. For the low- $T_{i}$ plasmas, the functions of $a(\rho)$ and $R / L_{T_{c}}$ are used as $a(\rho)=1.9+4.0 \times 10 \rho-3.4 \times 10^{2} \rho^{2}+1.1 \times 10^{3} \rho^{3}-1.1 \times 10^{3} \rho^{4}$ for $\rho \leq 0.5, a(\rho)=9.6 \times 10-5.7 \times 10^{2} \rho+1.3 \times 10^{3} \rho^{3}$ for $\rho>0.5$ and $R / L_{T c}=4.7-3.3 \times 10 \rho+1.5 \times 10^{2} \rho^{2}-2.0 \times$ $10^{2} \rho^{3}+8.5 \times 10 \rho^{4}$ in the dynamical transport simulation. In Fig. 4 (a), a jump around $\rho=0.5$ is found. This is because $\mathcal{L}$ is fitted with the linear function (6) in terms of $R / L_{T_{i}}$. In the region $\rho<0.5$, the values of $R / L_{T_{i}}$, which are close to the critical gradient, are taken, when $a(\rho)$ is evaluated. The stiffness is strong, when $R / L_{T_{i}}$ is close to $R / L_{T_{c}}$, so that the value of $a(\rho)$ becomes large. In the region $\rho>0.5$, we 

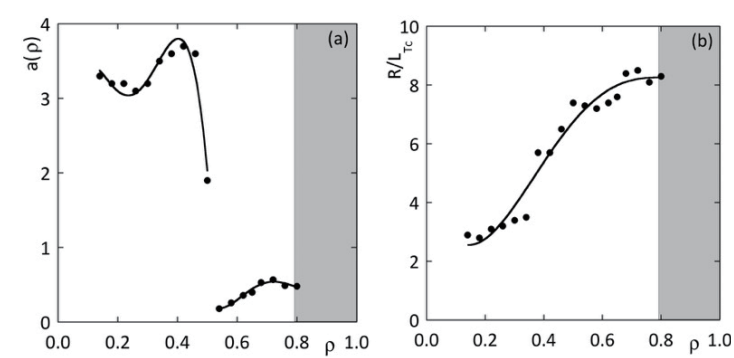

Fig. 4 The radial profiles of (a) a $\left(\rho\right.$ ) and (b) $R / L_{T_{c}}$ is shown with the filled circles for the low- $T_{i}$ plasmas. The curves represent the fitting function with respect to the radial position $\rho$.
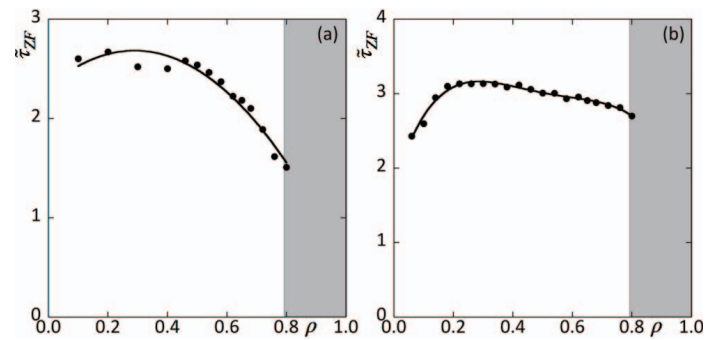

Fig. 5 The radial profiles of $\tilde{\tau}_{Z F}$ are shown with the filled circles for the (a) high- $T_{i}$ and (b) low- $T_{i}$ plasmas. The curves represent the fitting curve for the zonal flow decay time.

choose the values of $R / L_{T_{i}}$, which are far from the critical gradient, so that the value of $a(\rho)$ is small. The fitting of $\mathcal{L}$ with the other functions is for the future study.

The zonal flow decay time $\tilde{\tau}_{Z F}$ [21], which only depends on the magnetic field structure, is examined in the radial region $0.1 \leq \rho \leq 0.8$. The radial profile of the zonal flow decay time $\tilde{\tau}_{Z F}$ is shown in Fig. 5 (a) for the high- $T_{i}$ plasmas. The fitting function for the zonal flow decay time for the high- $T_{i}$ plasmas $\tilde{\tau}_{Z F}=2.3+2.5 \rho-4.3 \rho^{2}$ is used throughout the transport simulation for the high- $T_{i}$ plasmas, because the field configuration is fixed throughout the simulation. The radial profile of the zonal flow decay time $\tilde{\tau}_{Z F}$ is shown in Fig. 5 (b) for the low- $T_{i}$ plasmas. The fitting function for the zonal flow decay time for the low- $T_{i}$ plasmas $\tilde{\tau}_{Z F}=1.7+1.3 \times 10 \rho-4.4 \times 10 \rho^{2}+5.9 \times 10 \rho^{3}-$ $2.9 \times 10 \rho^{4}$ is used. Note that this modeling for $\mathcal{L}$ and the fitting function for the zonal flow decay time is applicable to the magnetic field structure used here and the fixed profiles of the density and the electron temperature. It is found that the additional modeling for $\mathcal{L}$ and $\tilde{\tau}_{Z F}$ reproduces the results of the reduced models for the turbulent electron and ion heat diffusivities, (1) and (2).

\section{Additional Modeling for Quasilinear Flux Models}

The quasilinear flux model for the particle and heat transport is shown in Eqs. (3) and (4). The ratios of the quasilinear flux models by use of the GKV-X code is de-

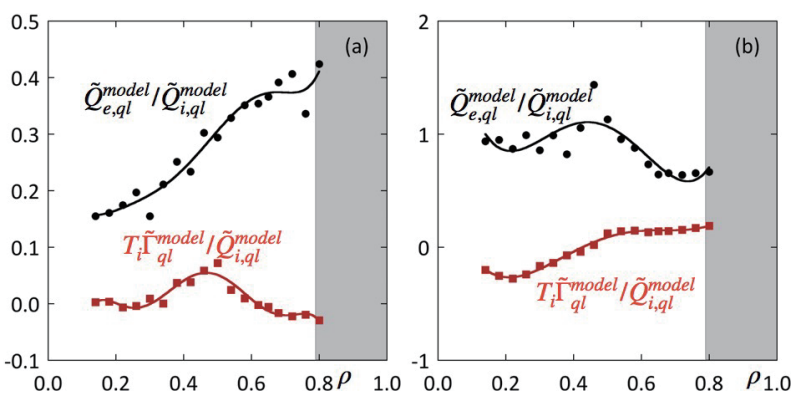

Fig. 6 For the (a) high- $T_{i}$ and (b) low- $T_{i}$ plasmas, the radial profiles of $\tilde{Q}_{e, q l}^{\text {model }} / \tilde{Q}_{i, q l}^{\text {model }}$ and $T_{i} \tilde{\Gamma}_{q l}^{\text {model }} / \tilde{Q}_{i, q l}^{\text {model }}$ are shown with the filled circles and the boxes, respectively. The solid curves represent the fitting functions for the radial profiles of $\tilde{Q}_{e, q l}^{\text {model }} / \tilde{Q}_{i, q l}^{\text {model }}$ and $T_{i} \tilde{\Gamma}_{q l}^{\text {model }} / \tilde{Q}_{i, q l}^{\text {model }}$.

termined by Eqs. (3) and (4). In the dynamical simulation, the ion heat flux is evaluated from the ion heat diffusivity model, because how to apply the ion heat diffusivity model to the transport code was already shown [24]. The radial profiles for the ratios of the quasilinear electron heat flux model to the quasilinear ion heat flux model for the high- $T_{i}$ and low- $T_{i}$ plasmas are shown with the filled circles in Fig. 6(a) and Fig. 6(b), respectively. Figure 6 (a) and Fig. 6(b) indicate the radial dependences of $T_{i} \tilde{\Gamma}_{q l}^{\text {model }} / \tilde{Q}_{i, q l}^{\text {model }}$ for the high- $T_{i}$ and low- $T_{i}$ plasmas with the boxes, respectively. The curves in Fig. 6 represent the fitting functions for the radial profiles of $\tilde{Q}_{e, q l}^{\text {model }} / \tilde{Q}_{i, q l}^{\text {model }}$ and $T_{i} \tilde{\Gamma}_{q l}^{\text {model }} / \tilde{Q}_{i, q l}^{\text {model }}$. The ratios of $\tilde{Q}_{e, q l}^{\text {model }} / \tilde{Q}_{i, q l}^{\text {model }}$ and $T_{i} \tilde{\Gamma}_{q l}^{\text {model }} / \tilde{Q}_{i, q l}^{\text {model }}$ are directly evaluated by the linear gyrokinetic simulation results. To obtain the values of $\tilde{Q}_{e, q l}^{\text {model }}$ and $\tilde{\Gamma}_{q l}^{\text {model }}$, the denominator $\tilde{Q}_{i, q l}^{\text {model }}$ is needed to be evaluated by the ion heat diffusivity model (2). The additional modeling for the quasilinear flux models is found to well reproduce the results of the quasilinear flux models for the turbulent electron heat and particle fluxes.

\section{Transport Analysis by Heat Diffusivity and Quasilinear Heat Flux Models}

Now, the transport dynamics of the electron or ion temperature is studied using the modeled heat diffusivities and the modeled quasilinear heat fluxes, when the integrated transport code, e.g., TASK3D [17] is performed. In this study, the time evolution of the density is not solved, because the analysis of the particle source for the experimental condition is not available in the TASK3D at present. First, the time evolution of $T_{i}$ or $T_{e}$ is studied in the high$T_{i}$ plasmas. The dynamics of the radial $T_{j}$ profile for the species $j$ is simulated by solving the diffusion equation as

$$
\frac{\partial}{\partial t}\left(\frac{3}{2} n T_{j}\right)=-\frac{1}{V^{\prime}} \frac{\partial}{\partial \rho}\left(V^{\prime} Q_{j}\right)+P_{h x j}+P_{h j},
$$

where $V$ is the plasma volume, $V^{\prime}=d V / d \rho$. Here, $P_{h x e}=-P_{h x}, P_{h x i}=P_{h x}$ and $P_{h x j}$ is the absorbed power 

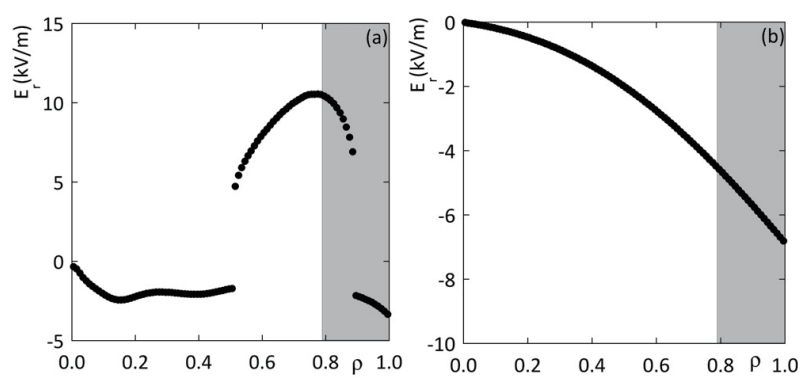

Fig. 7 The radial profiles of the electric field for the (a) high- $T_{i}$ and (b) low- $T_{i}$ plasmas is shown.

for the species $j$, where $P_{h x}$ is the heat exchange term. The heat flux $Q_{j}$ is set as $Q_{j}=-\left\langle|\nabla \rho|^{2}\right\rangle n\left(\chi_{j}+\chi_{j}^{N E O}\right) \partial T_{j} / \partial \rho$, where $\chi_{j}^{N E O}$ is the neoclassical diffusion coefficient for the species $j$ and $<>$ represents the magnetic surface average. The neoclassical diffusion coefficient is derived from DGN/LHD database with the low- $\beta$ limit $(\beta=0)$ [26].

For the dynamical simulation for the $T_{i}$ profile, the $T_{\mathrm{i}}$ profile of the experimental results for the high- $T_{i}$ plasmas is used for an initial state. The turbulent ion diffusivity $\chi_{i}$ is evaluated by substituting the fitting functions for $\mathcal{L}$ and $\tilde{\tau}_{Z F}$ in Eq. (2). The profile of the radial electric field $E_{r}$ is derived from the ambipolar condition at the initial plasma state. The positive $E_{r}$ is experimentally observed in the region $0.8<\rho<1.0$ for the high- $T_{i}$ plasma in the LHD of \#88343 [19]. Therefore, the largest positive radial electric field is chosen from three solutions of the ambipolar conditions, which is shown in Fig. 7 (a), where the three solutions of the ambipolar radial electric field are found in the radial region $0.52 \leq \rho \leq 0.89$. For the low- $T_{i}$ plasmas, one solution of the negative radial electric field for the ambipolar condition is obtained in Fig. 7 (b). The radial profiles of the density and the electron temperature are fixed, which are shown in Fig. 1. This is because the profiles of the density and the electron temperature are considered to be almost stationary in the experimental results [19]. Since the ion temperature profile is also considered to be nearly stationary in the experimental results [19], the radial profile of the ambipolar electric field is assumed to be fixed in this study. The value of the ion temperature is fixed at the initial state in the shaded region $0.80 \leq \rho \leq 1.0$, because the modeling for the turbulent heat diffusivities is performed in the region $0.14 \leq \rho \leq 0.80$. At the initial state, the radial profile for the absorbed power of ions, $P_{h i}$ is calculated using TASK3D and is fixed throughout the simulation and we use the fixed $q$ profile in Fig. 1 (c).

We show the simulation results for the kinetic electron response of the stationary ion temperature profile with the solid line in Fig. 8 (a), for comparison with the experimental result. The dashed and dotted lines indicate the profiles of $T_{i}$ for the simulation result in the adiabatic electron condition and the experimental results for the high- $T_{i}$ plasmas, respectively. In the adiabatic and kinetic electron conditions, the simulation results for the $T_{i}$ profiles show
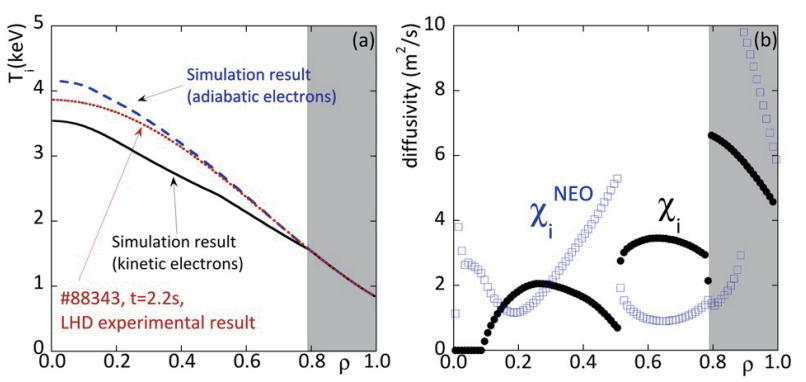

Fig. 8 (a) The $T_{i}$ profiles for the high- $T_{i}$ plasmas are shown. The solid line and the dashed line represent the ion temperatures for the simulation results in the kinetic and the adiabatic electron conditions, respectively. The dotted line indicates the experimental results at $t=2.2 \mathrm{~s}$ in the LHD. (b) The radial profiles of the ion neoclassical and turbulent diffusivities for the high- $T_{i}$ plasmas are shown. The filled circle and the blank box represent the neoclassical and turbulent diffusivities, respectively.

the good agreement with the experimental result. The ion energy flux at $\rho=0.65$ in the case with the experimental $T_{i}$ profile is about four times larger than that in the case with the simulated $T_{i}$ profile at a stationary state. The ion heat flux with the ion temperature gradient reduced by $20 \%$ from the experimental result is comparable to that by the stationary state for $T_{i}$ for the transport simulation result at $\rho=0.65$. Therefore, the stiffness is found to be strong in this case. The simulation result for the adiabatic electron condition shows better agreement with the experimental result than that for the kinetic electron response in this case. However, it is predicted that the simulation result for $T_{i}$ with the kinetic electron agrees with the experimental result better than that with the adiabatic electron for the high- $T_{i}$ plasmas in the other shots for the LHD [27]. Since the gyrokinetic transport models used in this article are proposed under the assumptions that there are still unintroduced effects, e.g., $\boldsymbol{E} \times \boldsymbol{B}$ shearing effects, which may improve the prediction of the $T_{i}$ profile, the simulation model should be improved. The stationary profiles of the turbulent and neoclassical diffusivities are also shown in Fig. 8 (b). The filled circles and the boxes represent the ion turbulent and neoclassical diffusivities. The ITG mode is destabilized in the radial region $0.10<\rho<0.8$. The turbulent transport is dominant compared with the neoclassical transport in the radial region $0.52 \leq \rho \leq 0.80$, where the positive electric field is chosen.

For the dynamical transport simulation for the $T_{e}$ profile, the radial $T_{\mathrm{e}}$ profile of the experimental results for the high- $T_{i}$ plasmas is used as an initial state. The turbulent electron diffusivity $\chi_{e}$ is evaluated by substituting the fitting functions for $\mathcal{L}$ and $\tilde{\tau}_{Z F}$ in Eq. (1). In another case, the turbulent electron diffusivity $\chi_{e}$ is estimated by the ratio of the quasilinear electron heat flux model to the quasilinear ion heat flux model, where the quasilinear ion heat flux model is estimated from the ion heat diffusivity model. Note that the change of the electron temperature 


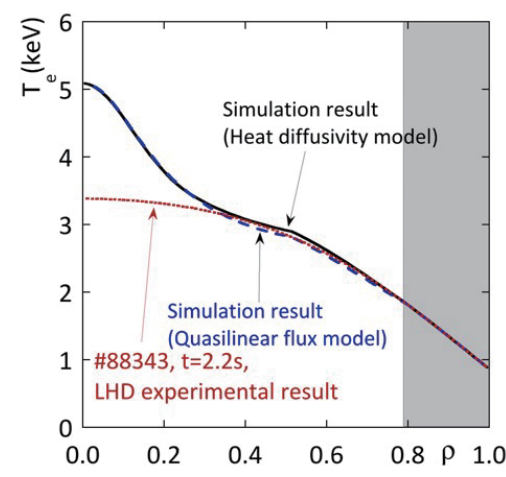

Fig. 9 The radial profiles of $T_{e}$ for the high- $T_{i}$ plasmas are shown. The solid line and the dashed line represent the electron temperature profiles for the simulation results, using the heat diffusivity model and the quasilinear flux model, respectively. The dotted line indicates the experimental results for the high- $T_{i}$ plasmas.

gradient in the time evolution is not included for the modeling for $\chi_{e}$ in this study. The radial profiles of the density and the ion temperature are fixed, which are shown in Fig. 1. The profile of the radial electric field in Fig. 7 (a) is also kept throughout the simulation. The value of the electron temperature is fixed at the initial state in the shaded region $0.80 \leq \rho \leq 1.0$. At the initial state, the radial profile for the absorbed power of electrons, $P_{h e}$ is calculated using TASK3D and is fixed. The simulation results of the stationary electron temperature profiles by using the electron heat diffusivity model and the quasilinear flux model are indicated with the solid line and the dashed line in Fig. 9, respectively. The dotted line represents the experimental results of the $T_{e}$ profile for the high- $T_{i}$ plasmas. The simulation results for the both cases agree well with the experimental results in the region $0.3<\rho<0.8$. However, the value of $T_{e}$ of the simulation result becomes larger than that of the experimental result in the region $0.0<\rho<0.3$, because the modeling for the turbulent transport by the linear simulation results is performed in the region $0.46<\rho<0.80$ and the time change of the electron temperature gradient is not included for the modeling for $\chi_{e}$ in this study. If the electron temperature gradient is included in the modeling for $\chi_{e}$, the electron heat diffusivity becomes larger even for the ITG mode and the value of the electron temperature is predicted to be smaller. In both cases of the simulation results, the electron neoclassical transport is dominant compared with the electron turbulent transport in the most radial region. The ITG mode becomes unstable in the region $\rho>0.2$.

Next, the time evolution of $T_{i}$ or $T_{e}$ is studied in the low- $T_{i}$ plasmas. The dynamical simulation for $T_{i}$ is performed, where the ion temperature profile shown in Fig. 2 (b) is used for the initial state. The radial profiles of $n$ and $T_{e}$ in Figs. 2 (a), (b), and the electric field in Fig. 7 (b) are fixed throughout the simulation. We show the simulation results of the stationary ion temperature profile with
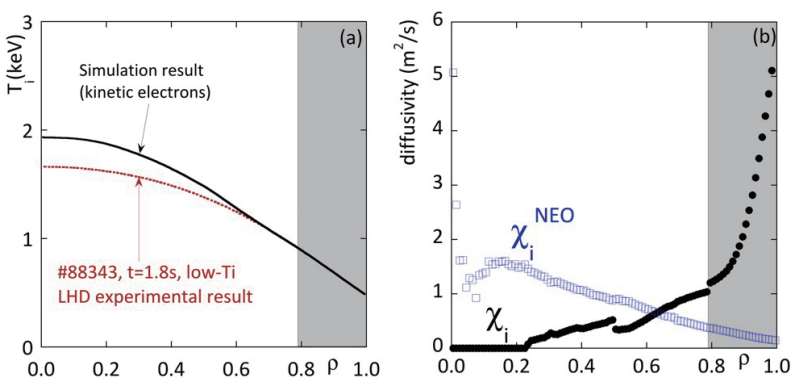

Fig. 10 (a) The $T_{i}$ profiles for the low- $T_{i}$ plasmas are shown. The solid line represents the $T_{i}$ profile for the simulation result. The dotted line indicates the experimental results at $t=1.8 \mathrm{~s}$ in the LHD. (b) The radial profiles of the ion neoclassical and turbulent diffusivities for the low- $T_{i}$ plasmas are shown. The filled circle and the box indicate the neoclassical and turbulent diffusivities, respectively.

the solid line in Fig. 10 (a), for comparing with the experimental result (the dotted line) for the low- $T_{i}$ plasmas. It is found that the simulation result for the low- $T_{i}$ does not contradict with experimental result. For the adiabatic electron condition, the ITG mode is unstable only in the region $\rho>0.6$ [28]. Therefore, the dynamical simulation for the adiabatic electron approximation is not performed in the low- $T_{i}$ plasmas. Figure 10 (b) indicates the radial profiles of the turbulent and neoclassical diffusivities. The ITG mode becomes unstable in the radial region $\rho>0.24$.

For the dynamical transport simulation for the $T_{e}$ profile, the $T_{\text {e }}$ profile of the experimental results for the low$T_{i}$ plasmas is used as an initial state. The radial profiles of the density and the ion temperature are fixed, which are shown in Fig. 2. The profile of the radial electric field in Fig. 7 (b) is also fixed throughout the simulation. The simulation results, using the electron heat diffusivity model and the quasilinear flux model, of the stationary electron temperature profiles are shown with the solid line and the dashed line in Fig. 11, respectively. The dotted line indicates the radial profile of $T_{e}$ of the experimental results for the low $-T_{i}$ plasmas. The $T_{e}$ profiles of the simulation results are found to be comparable to the experimental results in the low- $T_{i}$ plasmas. The electron temperature is larger than the experimental results except the $T_{e}$ values in the region $0.7<\rho<0.8$ by using quasilinear flux model. As explained above, it is predicted that the inclusion of the electron temperature steepening in the modeling for $\chi_{e}$ decreases the value of the electron temperature. Both the diffusivity and the quasilinear flux models for the electron heat transport predict almost the same $T_{e}$ profiles in Figs. 9 and 11. Therefore, it may be considered that the quasilinear flux model is not needed, because the quasilinear flux formulation is more complicated than the function of the diffusivity models. However, it is predicted that the electron heat diffusivity model can not be obtained for the flattened $T_{e}$ profile, which is different from Figs. 1 (b) and 2 (b). In fact, the particle diffusivity model is not shown for 


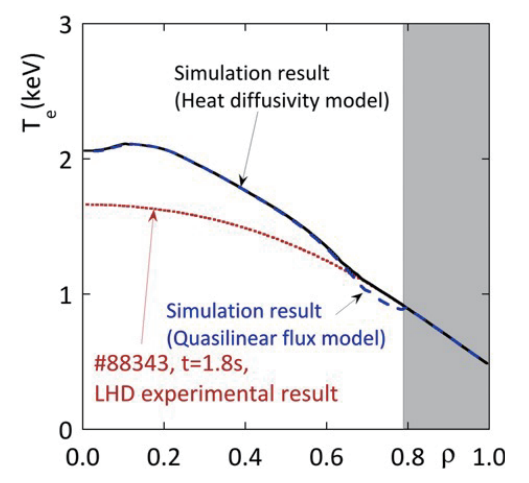

Fig. 11 The radial profiles of $T_{e}$ for the low- $T_{i}$ plasmas are shown. The solid line and the dashed line represent the electron temperatures for the simulation results, using the heat diffusivity model and the quasilinear flux model, respectively. The dotted line indicates the experimental results at $t=1.8 \mathrm{~s}$ in the LHD.

the flattened density profile which is typical for the LHD. This is because it is difficult to obtain the accurate values of the effective particle diffusivity. The quasilinear flux model for the particle transport can be obtained even for the flattened density profile [23]. The quasilinear flux formulation enables us to model the particle and heat transport for the flattened density and temperature profiles. The quasilinear models are not directly introduced to the transport simulation in this article. The quasilinear model for the electron heat transport is calculated by $\tilde{Q}_{e, q l}^{\text {model }} / \tilde{Q}_{i, q l}^{\text {model }}$, where $\tilde{Q}_{i, q l}^{\text {model }}$ is evaluated by the ion heat diffusivity model (2). The simulation study using the quasilinear models in this article is the preparatory one for the transport simulation, where the quasilinear flux models will be directly installed. In both cases of the simulation results, the electron neoclassical transport is larger than the turbulent transport in the radial region $\rho<0.7$. The ITG mode is unstable in the region $\rho>0.6$.

\section{Summary}

The dynamical transport simulation is performed for the turbulent transport by use of the heat diffusivity and the quasilinear flux models based on the gyrokinetic simulation results and the neoclassical transport by use of DGN/LHD database. The ion heat diffusivity model is used to evaluate the ion heat transport for the dynamical simulation of the ion temperature. In the kinetic electron condition, the $T_{i}$ profile of the simulation result is in agreement with that in the experimental result for the high- $T_{i}$ and low- $T_{i}$ plasmas in the LHD of the shot number 88343. The $T_{e}$ profile of the simulation results is found to be comparable to the experimental results for the high- $T_{i}$ and low$T_{i}$ plasmas, when the electron heat diffusivity model and electron quasilinear flux model are used. The inclusion of the electron temperature gradient for the ITG mode in the modeling of the electron heat transport is for the future study. The promising method [24] of a low compu- tational cost for adapting the gyrokinetic turbulent transport model to a transport code is used. It is found that the temperature profiles by the transport simulation, where the reduced models which reproduce the nonlinear simulation results are used, are comparable to the experimental results. Note that the heat diffusivity and the quasilinear models are shown by studying the ITG mode using the specified plasma profiles and field configurations in the high- and low- $T_{i} \# 88343$ plasmas. The heat diffusivity and the quasilinear flux models have been studied using the other plasma profiles and field configurations in the LHD [27]. The particle quasilinear flux model is needed for the transport simulation of the density evolution. The study of the particle source/sink in the LHD is under way. The dynamical simulation results for the density profile will be reported in another article.

This work was partly supported by the JSPS KAKENHI Grant Number, No. 16K06941, the NIFS Collaboration Research Programs, the NIFS18KNST129, the NIFS18KNXN363 (Plasma Simulator), the NIFS18KNTT045 and the Collaborative Research Program of Research Institute for Applied Mechanics, Kyushu University, 30FP-4. This work is carried out using the JFRS-1 supercomputer system at Computational Simulation Centre of International Fusion Energy Research Centre (IFERC-CSC) in Rokkasho Fusion Institute of QST (Project code: MTFHP).

[1] J.W. Connor and H.R. Wilson, Plasma Phys. Control. Fusion 36, 719 (1994).

[2] W. Horton, Turbulence Transport in Magnetized Plasmas, 2nd ed. (World Scientific Pub. Co Inc; 2017).

[3] X. Garbet et al., Nucl. Fusion 50, 0433002 (2010).

[4] F. Jenko and W. Dorland, Plasma Phys. Control. Fusion 43, A141 (2001).

[5] J. Candy and R.E. Waltz, J. Comput. Phys. 186, 545 (2003).

[6] T.-H. Watanabe, H. Sugama and S. Ferrando-Margalet, Nucl. Fusion 47, 1383 (2007).

[7] P. Xanthopoulos et al., Phys. Rev. Lett. 99, 035002 (2007).

[8] M. Nunami et al., Plasma Fusion Res. 6, 1403001 (2011).

[9] A. Ishizawa et al., Phys. Plasmas 21, 055905 (2014).

[10] M. Kotschenreuther et al., Phys. Plasmas 2, 2381 (1995).

[11] C. Holland et al., Phys. Plasmas 18, 056113 (2011).

[12] T.L. Rhodes et al., Nucl. Fusion 51, 063022 (2011).

[13] M. Nunami et al., Phys. Plasmas 19, 042504 (2012).

[14] M. Nunami, T.-H. Watanabe and H. Sugama, Phys. Plasmas 20, 092307 (2013).

[15] J. Candy et al., Phys. Plasmas 16, 060704 (2009).

[16] M. Barnes et al., Phys. Plasmas 17, 056109 (2010).

[17] M. Yokoyama et al., Plasma Fusion Res. 7, 2403011 (2012).

[18] T.-H. Watanabe and H. Sugama, Nucl. Fusion 46, 24 (2006).

[19] K. Tanaka et al., Plasma Fusion Res. 5, S2053 (2010).

[20] H. Sugama and T.-H. Watanabe, Phys. Plasmas 13, 012501 (2006).

[21] S. Ferrando-Margalet, H. Sugama and T.-H. Watanabe, Phys. Plasmas 14, 122505 (2007).

[22] S. Toda et al., Plasma Fusion Res. 12, 1303035 (2017). 
[23] S. Toda et al., Phys. Plasmas 26, 012510 (2019).

[24] S. Toda et al., J. Phys.: Conf. Ser. 561, 012020 (2014).

[25] T.-H. Watanabe, H. Sugama and S. Ferrando-Margalet, Phys. Rev. Lett. 100, 195002 (2008).

[26] A. Wakasa et al., Jpn. J. Appl. Phys. 46, 1157 (2007).
[27] S. Toda et al., 20th International Stellarator-Heliotron Workshop 5-9 October 2015, Greifswald, Germany, P1S224 (2015).

[28] A. Ishizawa et al., Nucl. Fusion 57, 066010 (2017). 